\title{
Backward transition radiation in the extreme ultraviolet region as a tool for the transverse beam profile diagnostic
}

\author{
L. G. Sukhikh, ${ }^{1, *}$ G. Kube, ${ }^{2}$ S. Bajt, ${ }^{2}$ W. Lauth, ${ }^{3}$ Yu. A. Popov, ${ }^{1, \dagger}$ and A. P. Potylitsyn ${ }^{1}$ \\ ${ }^{1}$ Tomsk Polytechnic University, Tomsk 634050, Russia \\ ${ }^{2}$ Deutsches Elektronen-Synchrotron (DESY), 22607 Hamburg, Germany \\ ${ }^{3}$ Institute of Nuclear Physics, Johannes Gutenberg University, 55128 Mainz, Germany
}

(Received 30 April 2013; revised manuscript received 24 September 2014; published 18 November 2014)

\begin{abstract}
The present article summarizes the results of two experiments which were performed to study the radiation properties of backward transition radiation (BTR) in the extreme ultraviolet (EUV) region. This wavelength region is of particular interest for transverse beam profile imaging, because the spatial resolution is improved as a result of the reduced contribution in the imaging process of the fundamental diffraction limit. In addition, the influence of coherent effects in the transition radiation emission process, which have been observed in the visible region, might be mitigated. The first experiment, dedicated to the investigation of the BTR angular characteristics, indicates that the radiation yield in the EUV region is higher than theoretically expected and that it is even comparable to the yield in the visible region. The second measurement was devoted to transverse beam profile imaging based on quasimonochromatic BTR, in both the EUV and the visible region, and is a proof-of-principle experiment demonstrating that EUV BTR is a suitable candidate for standard beam profile diagnostics.
\end{abstract}

DOI: 10.1103/PhysRevSTAB.17.112805

PACS numbers: 41.60.-m, 41.75.Ht

\section{INTRODUCTION}

Transition radiation is emitted when a charged particle crosses the boundary between two media with different optical properties. The radiation is generated in an instantaneous process directly at the screen boundary, and it has a lobe-shape pattern with a characteristic opening angle as defined by the beam energy. Neglecting coherent effects, transition radiation has a linear response and a rather high light output that makes its application suitable for subnanocoulomb particle beam diagnostics in modern linear accelerators. Because of practical reasons, the visible spectral part of this radiation (so-called optical transition radiation or OTR) which is emitted in the direction of the specular reflection is typically used for transverse beam profile imaging [1]. Transition radiation emitted in the backward direction is referred to as backward transition radiation (BTR) throughout this paper independent of the photon energy. In an intuitive picture, the generation of BTR can be simply considered as a reflection of the electromagnetic field of the particle by the target surface; i.e., the source is represented by pseudophotons that are converted to real photons through the momentum transfer from the target. The working principle of an OTR beam

\footnotetext{
*Sukhikh@tpu.ru

Present address: Joint Institute for Nuclear Research, Dubna, Russia.

Published by the American Physical Society under the terms of the Creative Commons Attribution 3.0 License. Further distribution of this work must maintain attribution to the author $(s)$ and the published article's title, journal citation, and DOI.
}

profile monitor has the beam spot imaged via OTR onto a spatial resolving detector by using a conventional optical system. Using such a technique, Ross et al. obtained a beam image with a resolution of about $2 \mu \mathrm{m}$ [2]. Unfortunately, there are physical limitations that make this method ineffective for reliable diagnostics in modern accelerators such as linac-based free electron lasers (FELs).

The experience from these FELs shows that OTR diagnostics might fail because of coherent effects in the OTR emission process. In the case of full coherent emission, the radiation intensity scales quadratically with the bunch population; thus, the profile of the light spot imaged onto the spatial resolving detector does not represent the transverse beam profile. Although electron bunch lengths in modern FELs are significantly larger than visible wavelengths, some unstable microstructures radiating coherently might appear in the bunch. In the case of full coherent OTR generation, the image of an electron beam with a Gaussian transverse profile has a doughnut structure. This was observed, for example, at the Linac Coherent Light Source (LCLS) (SLAC, USA) [3] and at Free electron laser in Hamburg (FLASH) (DESY, Germany) [4]. As a consequence, a new reliable transverse beam profile diagnostic is required for the fourth-generation $\mathrm{x}$-ray sources, e.g., the European X-FEL, which is currently under construction at DESY [5]. At first glance, a method to overcome the influence of coherent emission is to use scintillators instead of OTR screens because of the stochastic nature of the luminescence process. However, transition radiation is also emitted at the boundary between the vacuum and scintillator; thus, coherent radiation in the visible region can still appear. Based on the isotropic light emission from 
scintillators and the resolution studies in Ref. [6], it was proposed to spatially separate scintillation and BTR light by a proper choice of the monitor geometry $[7,8]$, thus avoiding the contribution of intense coherent radiation in profile measurements [9]. Screen monitors utilizing the principle of spatial suppression were developed for the European $\mathrm{X}-\mathrm{FEL}$ [10] and have been in operation at FLASH2 (DESY, Germany) for about half a year. A similar monitor developed at the Paul Scherrer Institute (Switzerland) was recently tested at the LCLS [11]. Anyhow, a resolution of the scintillator-based profile monitor is limited due to intrinsic material properties and observation geometry $[8,12,13]$.

A second physical limitation is the fundamental resolution in the imaging process that is usually discussed in terms of the point-spread function (PSF). The PSF describes the image of a point source, i.e., the image of a single electron in the case of OTR generation. The OTR PSF was investigated for the first time by Castellano and Verzilov [14], and later in more detail by Potylitsyn [15], Xiang and Huang [16], and Kube [17]. It was shown that the PSF has a double-lobe structure defined by the radiation wavelength, the acceptance of the optical system, and the alignment accuracy. In principle, minimum beam sizes of about 1-2 $\mu \mathrm{m}$ (rms) could be measured based on the standard OTR technique with a wavelength of $400 \mathrm{~nm}$ and a reasonable optical system. Ross et al. measured a vertical beam size as small as $5 \mu \mathrm{m}$ (rms) without observing an influence of PSF [2]. However, a recent experiment by Karataev et al. showed the PSF-dominated beam images which were measured at the Accelerator Test Facility II at the High Energy Accelerator Research Organization KEK (Tsukuba, Japan) [18]. The observed double-lobe structures were significantly wider than those predicted theoretically. As a result, beam images were significantly distorted; i.e., non-Gaussian beam shapes were imaged even for beams with vertical sizes as large as $4 \mu \mathrm{m}$ (rms).

A possibility to overcome both limitations, i.e., to avoid coherent emission at a given wavelength and to improve the spatial resolution in the imaging process, is to decrease the wavelength of observation $\lambda$. The idea to use BTR in the extreme ultraviolet (EUV) region at $\lambda \simeq 20 \mathrm{~nm}$ was first published in Ref. [19].

This paper presents the results of an experimental study to demonstrate the applicability of EUV BTR for electron beam profile diagnostics. While some of the results were previously reported [20-22], a comprehensive summary is given cumulating in the description of the proof-ofprinciple experiment for EUV BTR beam imaging. The paper is organized as follows: in Sec. II, a theoretical model to calculate the BTR characteristics in the EUV spectral region is described together with the experimental investigation of the transition radiation spatial distribution. Results of transverse beam profile imaging in the EUV and the optical spectral region are presented in Sec. III, and a summary and conclusions are given in Sec. IV.

\section{INVESTIGATION OF THE EUV BTR ANGULAR DISTRIBUTION}

\section{A. Theoretical model}

The problem is formulated as follows: an electron with charge $e$ travels with the reduced velocity $\beta=v / c$ and Lorentz factor $\gamma=\left(1-\beta^{2}\right)^{-1 / 2}$ along the $z$ axis as indicated in Fig. 1. The target is assumed to extend to infinity in the transverse $(x y)$ plane. This assumption means in practice that transverse dimensions of the target are at least a few times larger than the effective electron field radius, which is of the order of $\gamma \lambda$ for a given photon wavelength $\lambda$. The target is a spatially homogeneous material with permittivity $\varepsilon(\hbar \omega)$, which depends only on the photon energy $\hbar \omega=2 \pi \hbar c / \lambda$, and is tilted by an angle $\alpha$ with respect to the electron trajectory. The electron traverses the target, resulting in BTR in a wide spectral range, emitted in the direction of the specular reflection and measured with a spatially resolving detector.

The assumption of an infinite target allows one to use a simple analytical model of BTR generated by an ideally reflecting target [23]. In both the far-field approximation and high electron energy assumption $(\gamma \gg 1)$, the radiation field $\mathbf{E}\left(\theta_{x}, \theta_{y}\right)$ depends only on the projection angles $\theta_{x}=\frac{X}{L}$ (in the $x z$ plane, $L$ is the target-detector distance) and $\theta_{y}=\frac{Y}{L}$ (in the plane defined by the $y$ axis and the specular reflection direction) that are measured with respect to the direction of specular reflection (see Fig. 1) and does not depend on the target tilt angle $\alpha$. The radiation field is given by the following expression:

$$
\begin{aligned}
\mathbf{E}\left(\theta_{x}, \theta_{y}\right)= & \frac{e \beta}{\pi c} \frac{1}{1-\beta^{2} \cos ^{2} \theta_{x} \cos ^{2} \theta_{y}} \frac{e^{i \mathbf{k} \cdot \mathbf{r}}}{r} \\
& \times\left\{\cos \theta_{x} \sin \theta_{x} \cos \theta_{y},-\cos ^{2} \theta_{x} \cos \theta_{y} \sin \theta_{y},\right. \\
& \left.-\left(\sin ^{2} \theta_{x}+\cos ^{2} \theta_{x} \sin ^{2} \theta_{y}\right)\right\} .
\end{aligned}
$$

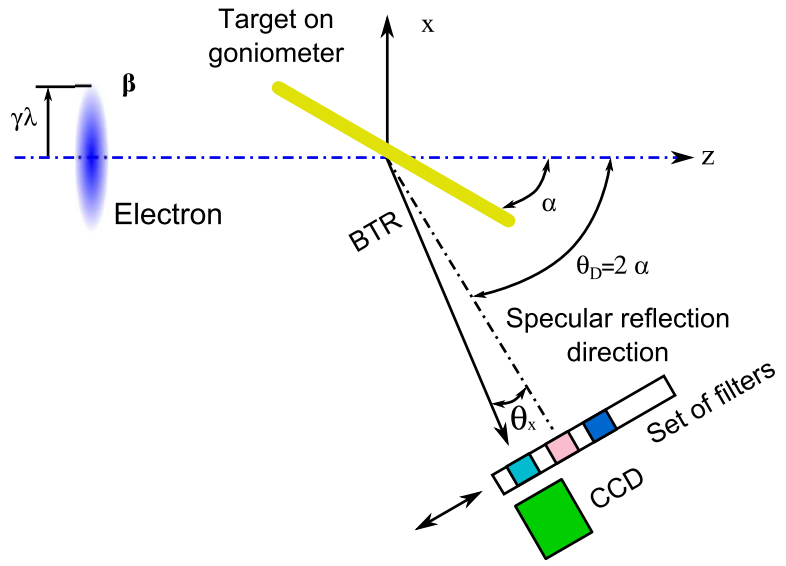

FIG. 1. Sketch of the setup for the experiment dedicated to the investigation of EUV BTR. 
Here $\mathbf{k}$ is the wave vector of an emitted BTR photon, and $\mathbf{r}$ designates the detector location.

Both real optical properties of the target and its tilt are taken into account by using the Fresnel reflection coefficients $R_{\sigma}(\alpha, \hbar \omega)$ and $R_{\pi}(\alpha, \hbar \omega)$, which depend on both the photon energy $\hbar \omega$ and on the target tilt angle $\alpha$. The spectral-angular radiation intensity can be expressed as

$$
\begin{aligned}
& \frac{d^{2} W\left(\theta_{x}, \theta_{y}, \hbar \omega\right)}{\hbar d \omega d \Omega} \\
& =\frac{c r^{2}}{\hbar}\left\{R_{\sigma}(\alpha, \hbar \omega)\left|E_{y}\left(\theta_{x}, \theta_{y}\right)\right|^{2}\right. \\
& \left.\quad+R_{\pi}(\alpha, \hbar \omega)\left[\left|E_{x}\left(\theta_{x}, \theta_{y}\right)\right|^{2}+\left|E_{z}\left(\theta_{x}, \theta_{y}\right)\right|^{2}\right]\right\}
\end{aligned}
$$

with the Fresnel coefficients written in the form

$$
\begin{aligned}
& R_{\sigma}(\alpha, \hbar \omega)=\left|\frac{\cos (\pi / 2-\alpha)-\sqrt{\varepsilon(\hbar \omega)-\sin ^{2}(\pi / 2-\alpha)}}{\cos (\pi / 2-\alpha)+\sqrt{\varepsilon(\hbar \omega)-\sin ^{2}(\pi / 2-\alpha)}}\right|^{2}, \\
& R_{\pi}(\alpha, \hbar \omega)=\left|\frac{\varepsilon \cos (\pi / 2-\alpha)-\sqrt{\varepsilon(\hbar \omega)-\sin ^{2}(\pi / 2-\alpha)}}{\varepsilon \cos (\pi / 2-\alpha)+\sqrt{\varepsilon(\hbar \omega)-\sin ^{2}(\pi / 2-\alpha)}}\right|^{2} .
\end{aligned}
$$

In this picture, a relativistic electron is described as a source of virtual photons and BTR generation is treated as a reflection of the virtual photon flux by the target, which is considered to be a mirror characterized by its Fresnel coefficients.

Molybdenum was chosen for the target material in the experiment due to its high reflectivity in the photon energy range $\hbar \omega=30 \ldots 100 \mathrm{eV}$ for grazing incidence angles $\alpha \leq 30^{\circ}$. The permittivity values for molybdenum in the energy range from 1 to $30 \mathrm{eV}$ were taken from Ref. [24]; the reflection coefficients of the target in the range from 30 to $150 \mathrm{eV}$ were obtained from the database in Ref. [25].

Figure 2 shows a calculated angular intensity distribution of monochromatic BTR $(\lambda=20 \mathrm{~nm})$ generated by $10^{10}$ electrons with $855 \mathrm{MeV}$ energy in the molybdenum target with a tilt angle $\alpha=28.1^{\circ}$. One can see in Fig. 2 that the BTR distribution is asymmetric due to a difference in the Fresnel coefficients.

Because of the finite distance between the target and detector in the experiment, only spatial and no angular BTR distributions were measured. Therefore, a convolution of the angular beam divergence and beam size has to be taken into account in Eq. (2) in order to compare with the experimental results. In the convolution procedure, two obvious facts were taken into account: (i) $\Delta \theta_{x, y}^{e}=2 \Delta \theta_{x, y}^{p h}$ ( $\Delta \theta_{x, y}^{e}$ is the electron angle with respect to the beam axis, and $\Delta \theta_{x, y}^{p h}$ is the additional photon emission angle with respect to $\theta_{x, y}$ ), and (ii) $\Delta_{x, y}^{e}=\Delta_{x, y}^{p h}\left(\Delta_{x, y}^{e}\right.$ is the shift of an electron with respect to the beam axis, and $\Delta_{x, y}^{p h}$ is the shift of the BTR distribution generated by the electron in the detector plane).

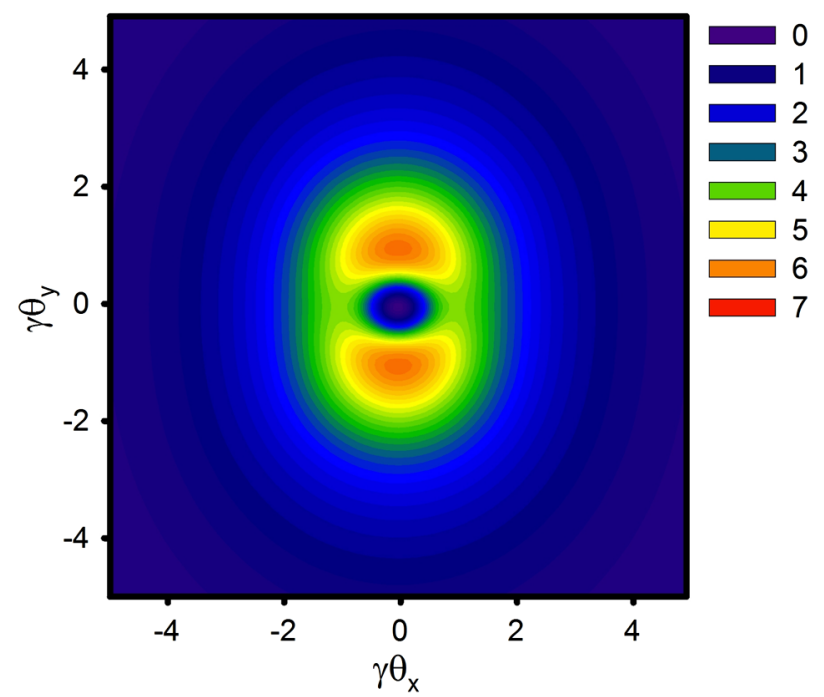

FIG. 2. Angular intensity distribution of BTR generated by $10^{10}$ electrons in the molybdenum target with the tilt angle $\alpha=28.1^{\circ}$. The electron energy is $855 \mathrm{MeV}$, and the radiation wavelength is $20 \mathrm{~nm}$.

\section{B. Experimental setup}

The experiment was carried out by using the $855 \mathrm{MeV}$ electron beam of the Mainz Microtron (MAMI) (Institute of Nuclear Physics, University of Mainz, Germany) [26]. This beam has a rather low beam emittance with typical rms values of about $\varepsilon_{y}^{e}=1 \mathrm{~nm}$ rad and $\varepsilon_{x}^{e}=9 \mathrm{~nm}$ rad. Figure 1 shows the schematic view of the experimental setup. The electron beam interacted with the target, which was tilted by an angle $\alpha$ with respect to the beam axis. The BTR spatial distributions, generated in both the visible and EUV region, were recorded with a CCD camera which was installed at a distance of $L=1010 \mathrm{~mm}$ from the target. Wavelength selection was performed by the choice of filters which could be inserted in front of the CCD.

The BTR targets were produced by the company Incoatec (Germany) [27] using magnetron sputtering technology. They consisted of a $50 \mathrm{~nm}$ thick molybdenum layer which was evaporated onto a $0.5 \mathrm{~mm}$ thick silicon substrate with dimensions of $40 \mathrm{~mm} \times 10 \mathrm{~mm}$. According to the company, the averaged (rms) surface roughness is better than $0.5 \mathrm{~nm}$ which was measured with a spatial resolution of about $1 \mathrm{~mm}$. The target was mounted onto remotely controlled goniometric stages for a precise target alignment with respect to the beam axis. Two geometries were used during the course of the experiment: the target tilt angle was either $\alpha=28.1^{\circ}$ or $\alpha=67.5^{\circ}$. The choice of tilt angle reflects the fact that the photon reflectivity in the EUV region depends significantly on the incidence angle. The first geometry was chosen to generate sufficient photons for EUV radiation detection, the second to detect a strong decrease in the number of EUV photons.

The angular distributions were recorded with a scientificgrade CCD camera ANDOR DO434 BN [28] with 


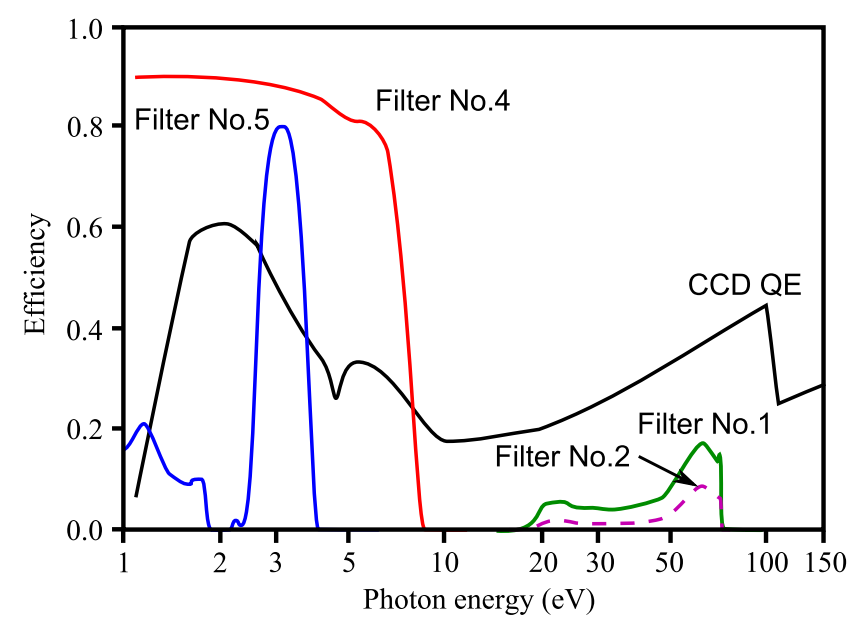

FIG. 3. CCD quantum efficiency (QE) and transmission efficiencies for the filters from Table I as a function of the photon energy. QE, black curve; filter No. 1, green curve; No. 2, purple curve; No. 4, red curve; No. 5, blue curve. The transmission efficiency of filter No. 3 was omitted for clarity.

$1024 \times 1024$ pixels and a pixel size of $13 \times 13 \mu \mathrm{m}^{2}$. The special feature of this 16-bit in-vacuum CCD camera is the high sensitivity in the photon energy range from $1 \mathrm{eV}$ up to $10 \mathrm{keV}$ due to the use of a back-illuminated CCD low-noise sensor chip from Marconi CCD47-10 [29] without an antireflection coating. Figure 3 shows the CCD quantum efficiency of the CCD as a function of the photon energy, as published by the manufacturer. In order to decrease the dark current, the camera was cooled to $-40{ }^{\circ} \mathrm{C}$ during the course of the experiment.

A remotely controlled filter set mounted onto a motorized holder was installed in front of the CCD. The set with the filter parameters summarized in Table I consisted of three aluminum foils of different thicknesses, a fused silica (FS) filter, and an optical bandpass (BP) filter with a central wavelength of $\lambda_{c}=400 \mathrm{~nm}$. In addition, it was possible to position the holder for free radiation passage and for complete radiation absorption by a $1 \mathrm{~mm}$ thick aluminum plate. The transmission efficiencies of selected filters are plotted in Fig. 3. The BP and the FS filter data were obtained from the manufacturers, the aluminum foil data from the database in Ref. [25]. The aluminum foils are opaque for visible light but allow radiation transmission

TABLE I. Filter characteristics.

\begin{tabular}{ll}
\hline \hline No. & \multicolumn{1}{c}{ Type } \\
\hline 1 & Al foil, thickness $1.3 \mu \mathrm{m}$ (two layers of $0.65 \mu \mathrm{m})$ \\
2 & Al foil, thickness $1.8 \mu \mathrm{m}$ \\
3 & Al foil, thickness $2.45 \mu \mathrm{m}$ \\
4 & Fused silica \\
5 & Optical bandpass filter with $\lambda_{c}=400 \mathrm{~nm}$ \\
& $($ FWHM $=140 \mathrm{~nm})$ \\
\hline \hline
\end{tabular}

TABLE II. Beam sizes (FWHM) at the target location as measured with the wire scanner. Beam configuration No. 3 was obtained with all quadrupoles switched off and was used only for the measurement with target tilt angle $\alpha=67.5^{\circ}$.

\begin{tabular}{lcc}
\hline \hline No. & Vert. FWHM $(\mu \mathrm{m})$ & Horiz. FWHM $(\mu \mathrm{m}) \%$ \\
\hline 1 & 64 & 533 \\
2 & 542 & 365 \\
3 & 247 & 6111 \\
\hline \hline
\end{tabular}

in the EUV region due to the x-ray absorption $L$ edge at $\hbar \omega_{L} \approx 72 \mathrm{eV}$; see Fig. 3 .

The quasicontinuous beam of the $855 \mathrm{MeV}$ racetrack microtron was operated in macropulse mode with a pulse duration of $0.8 \mathrm{~s}$ and with $3 \mathrm{~s}$ gaps in order to generate enough photons in the pulse and to allow for a full frame readout of the CCD during the gaps. For the investigation of the EUV BTR angular distribution, the average beam current was $52 \mathrm{nA}$, which corresponds to $2.6 \times 10^{11}$ particles per pulse (pulse duration $0.8 \mathrm{~s}$ ). The images obtained with the aluminum foil filters were accumulated over 200 pulses, the ones obtained with the optical filters over 20 pulses. For background subtraction, images were accumulated over 100 pulse trains without a beam.

For the experiment, three different beam sizes were used; see Table II. In order to adjust the beam configuration, the focusing strengths of the upstream quadrupoles in front of the experimental chamber were modified and the beam sizes were measured with a wire scanner at the location of the BTR target.

\section{Measurements and results}

For a comparison of the measured intensities under different experimental conditions, all measurements presented in this section were normalized to a total number $N_{e}=10^{10}$ electrons, which is a reasonable bunch population for modern accelerators. The parameter accessible in the measurement is the number of CCD counts per pixel $N^{\mathrm{pix}}\left(\theta_{x}, \theta_{y}\right)$. In order to compare the measured radiation yield with theoretical predictions, Eq. (2) was adapted to the experimental conditions, resulting in

$$
\begin{aligned}
N^{\mathrm{pix}}\left(\theta_{x}, \theta_{y}\right)= & N_{e} \int \frac{1}{\hbar \omega} \frac{d^{2} W\left(\theta_{x}, \theta_{y}, \hbar \omega\right)}{\hbar d \omega d \Omega} T_{f}^{i}(\hbar \omega) \\
& \times Q_{C C D}(\hbar \omega) d(\hbar \omega) d \Omega .
\end{aligned}
$$

$T_{f}^{i}(\hbar \omega)$ is the transmission coefficient of the $i$ th filter according to Table $3, Q_{C C D}(\hbar \omega)$ is the quantum efficiency of the CCD detector from Ref. [28], and $d \Omega$ is the solid angle as seen by a single CCD pixel.

Figure 4 shows examples of measured BTR spatial distributions in both the EUV and the visible spectral regions, which were obtained for electron beam 

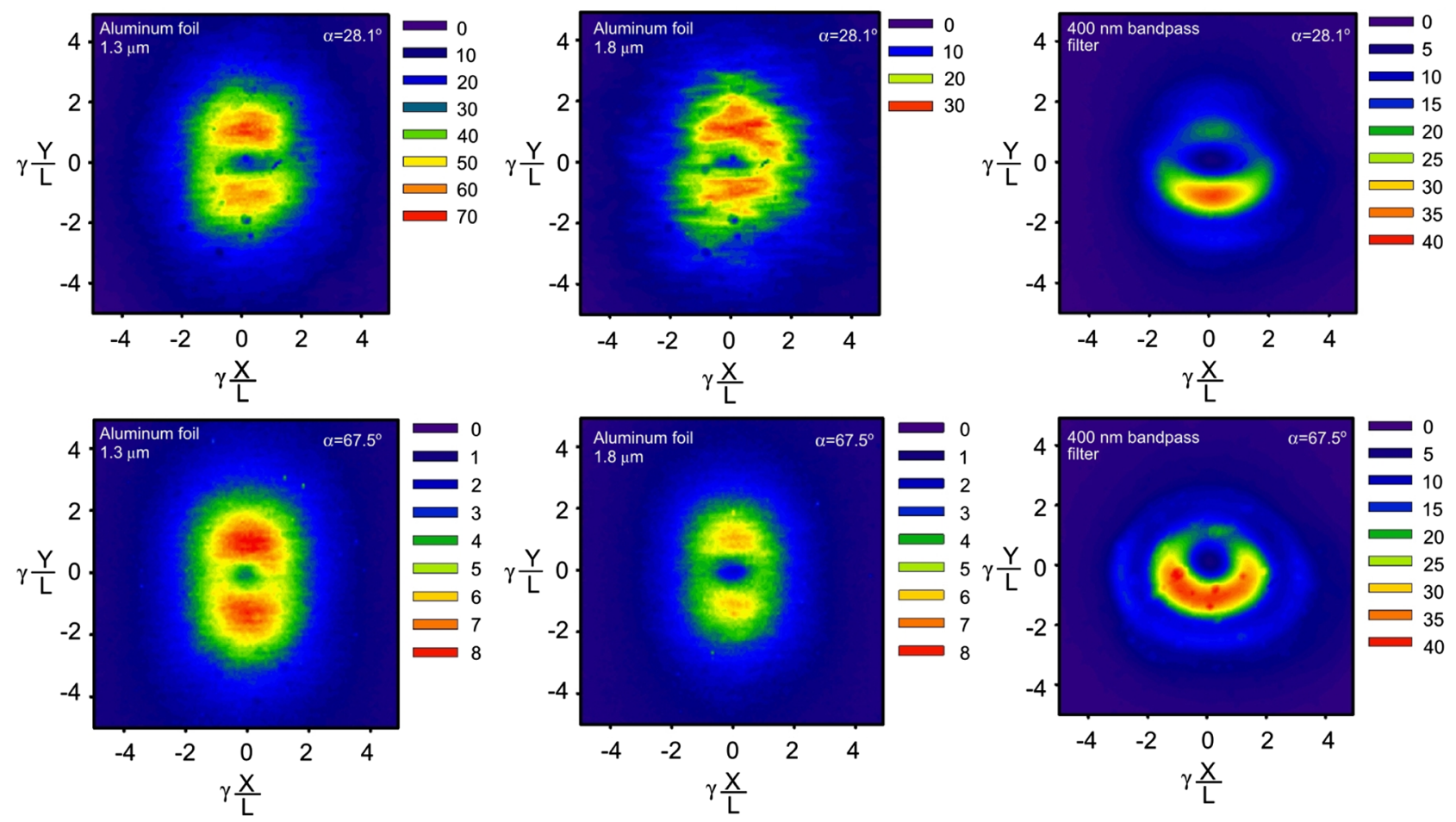

FIG. 4. Examples of BTR spatial distributions in the EUV and optical spectral region, measured with electron beam configuration No. 1; see Table II. Upper row: measurements for a target tilt angle $\alpha=28.1^{\circ}$; lower row: measurements for $\alpha=67.5^{\circ}$. Filters in use (from left to right): Al foil 1.3 and $1.8 \mu \mathrm{m}$ for observation of EUV radiation and BP filter $\lambda_{c}=400 \mathrm{~nm}$ for observation of visible light.

configuration No. 1; see Table II. Here it can be seen that all distributions have a shape similar to the shape of BTR.

However, BTR spatial distributions in the visible spectral region show an up-down asymmetry and even an oscillatory structure for the beam configurations No. 1 and No. 2. As can be seen in Fig. 4, the shapes differ significantly from that in the EUV region. The interpretation is that synchrotron radiation originating from the upstream quadrupole magnets interferes with BTR in the visible region and causes the observed structures in the BTR spatial distributions. The assumption of background from quadrupole synchrotron radiation is supported by the observation that for beam configuration No. 3 (quadrupole magnets switched off) a symmetrical spatial distribution without oscillatory structures was measured in both wavelength regions. Furthermore, the observation of an intense quadrupole synchrotron radiation background in the visible spectral region at MAMI was reported in Ref. [30].

The shape of the BTR spatial distributions measured in the EUV region for both geometries are close to the calculations; cf. Fig 2. A comparison of the measured BTR intensities for different geometries shows that the intensity of EUV BTR depends significantly on the target tilt angle as expected from the Fresnel coefficients Eq. (3), while the intensity in the visible region is nearly constant. It is interesting to note that the EUV BTR intensity for grazing incidence geometry $\left(\alpha=28.1^{\circ}\right)$ and with the $1.3 \mu \mathrm{m}$ aluminum filter is about a factor of 2 higher than that of optical BTR after the BP filter.

Figure 5 shows vertical cuts through the center of the measured spatial distributions, i.e., at $\gamma X / L=0$ in Fig. 4. The data are shown for beam No. 1 , the $1.3 \mu \mathrm{m}$ thick Al foil filter, and both target tilt angles. In the case of $\alpha=28.1^{\circ}$ [Fig. 5(a)], the signal-to-noise ratio is worse than for $\alpha=67.5^{\circ}$ [Fig. 5(b)]. This observation reflects the fact that the effective target thickness for the smaller tilt angle is nearly double that with $\alpha=67.5^{\circ}$. In addition, the calculated intensity distributions are shown, taking into account the influence of beam size and beam angular divergence which was obtained from the beam emittance. For better comparison, the theoretical curves are scaled by intensity. The difference in the scaling factors for the tilt angles suggests a nonsatisfactory description by the Fresnel coefficients Eq. (3), caused e.g., by an additional oxide layer on the target surface. However, for beam diagnostic purposes the absolute intensity is not a key issue. More significantly, the positions of the measured maxima in the angular distribution correspond well with the predictions. The smearing out of the central minimum might be caused by radiation background in the EUV region from upstream magnets.

It may thus be concluded that the agreement of the experimental data and the theoretical calculations with respect to the shape of the radiation spatial distributions 

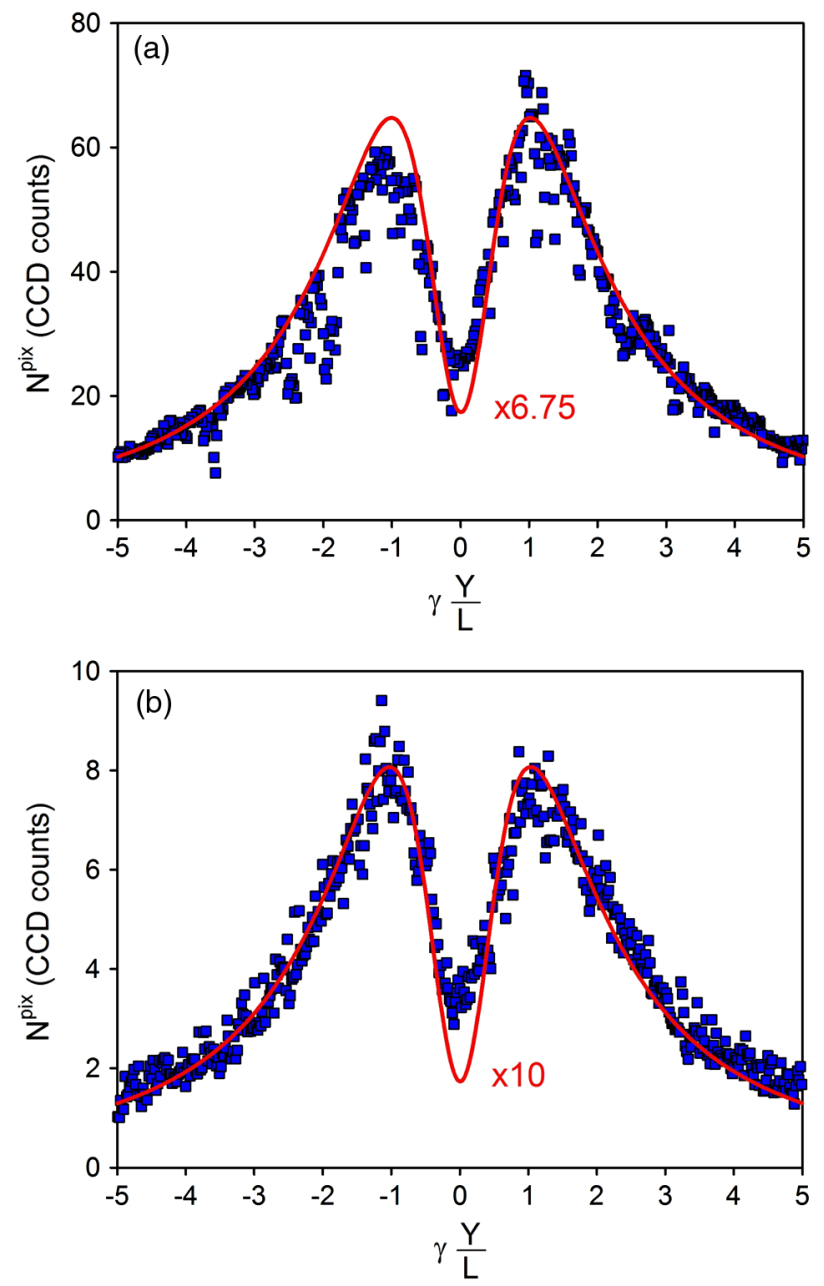

FIG. 5. Vertical cuts of calculated (red solid line) and measured (blue squares) BTR spatial distributions at $\gamma X / L=0$ for beam No. 1, Al foil filter $1.3 \mu \mathrm{m}$, and $\alpha=28.1^{\circ}$ (a), $\alpha=67.5^{\circ}$ (b). To aid the comparison, the theoretical curves are scaled by intensity. The scaling factor is 6.75 for $\alpha=28.1^{\circ}$ and 10 for $\alpha=67.5^{\circ}$.

confirms the nature of the observed radiation to be BTR in the EUV region.

\section{BEAM IMAGING BASED ON EUV BTR}

\section{A. Experimental setup}

Figure 6 shows a schematic view of the setup of the transverse beam profile imaging experiment in the EUV region, which was also carried out by using the $855 \mathrm{MeV}$ beam of the Mainz Microtron MAMI. The electron beam interacted with the target and generated BTR in a wide spectral range. The BTR was focused and additionally monochromatized in the EUV spectral region by a spherical multilayer mirror, and the beam spot was imaged onto the chip of the CCD camera used in the previous experiment. The distance from the target to the mirror was about $282 \mathrm{~mm}$ and from the mirror to the CCD $2535 \mathrm{~mm}$, resulting in an optical magnification of 9. Wavelength

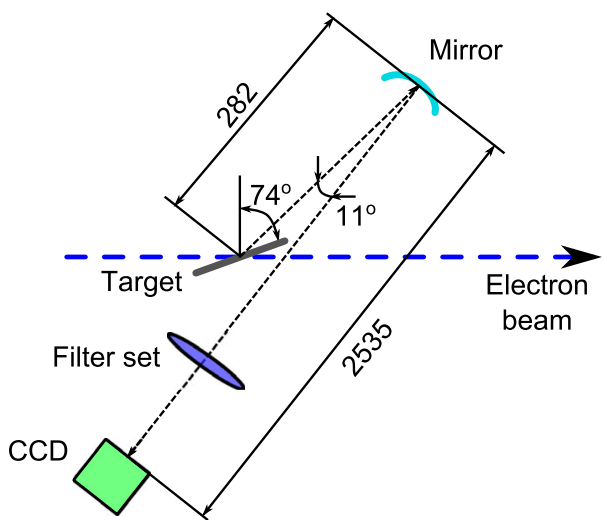

FIG. 6. Scheme of the beam imaging experiment setup.

selection could be performed by the filter set listed in Table I in order to selectively detect the visible or EUV part of the spectrum.

The BTR target also produced by the Incoatec company [27] consisted of a $50 \mathrm{~nm}$ thick molybdenum layer with surface roughness better than $0.5 \mathrm{~nm}$, which was evaporated onto a $0.7 \mathrm{~mm}$ thick silicon substrate with dimensions $50 \mathrm{~mm} \times 50 \mathrm{~mm}$. The target was mounted onto remotely controlled goniometric stages, which allowed a rotational motion and two linear motions along and across the beam axis for precise alignment with respect to the beam axis. The target surface normal was tilted by $\alpha=74^{\circ}$ with respect to the beam axis.

The spherical multilayer $\mathrm{Mo} / \mathrm{Si}$ mirror with $25.4 \mathrm{~mm}$ diameter and $250 \mathrm{~mm}$ focal distance used for the BTR focusing was designed and manufactured at DESY. The mirror was mounted onto remotely controlled rotational stages, thus allowing pitch and yaw motions. Figure 7 shows its reflectivity curve measured at the Physikalisch Technische Bundesanstalt (PTB), the German national metrology institute beam line of Bessy II (Berlin, Germany). At the central wavelength of $19.55 \mathrm{~nm}(64 \mathrm{eV})$, the peak mirror reflectivity

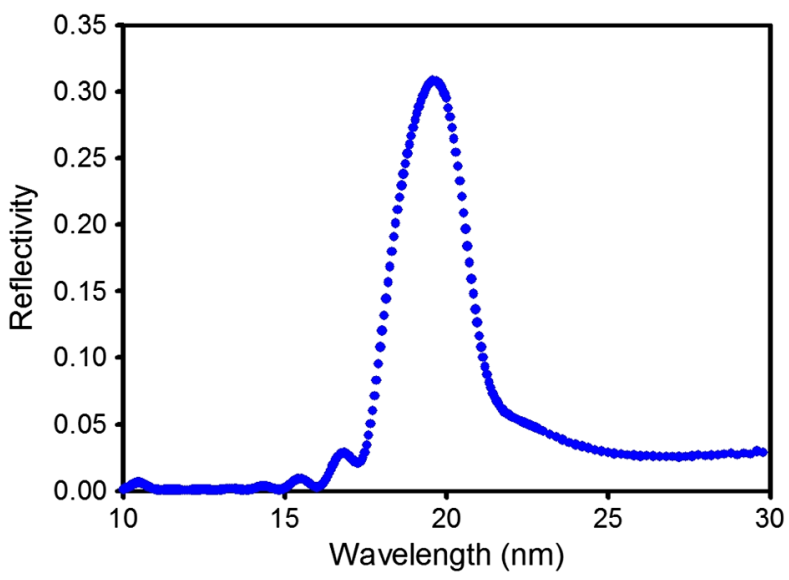

FIG. 7. Reflectivity curve of the spherical Mo/Si multilayer mirror. 


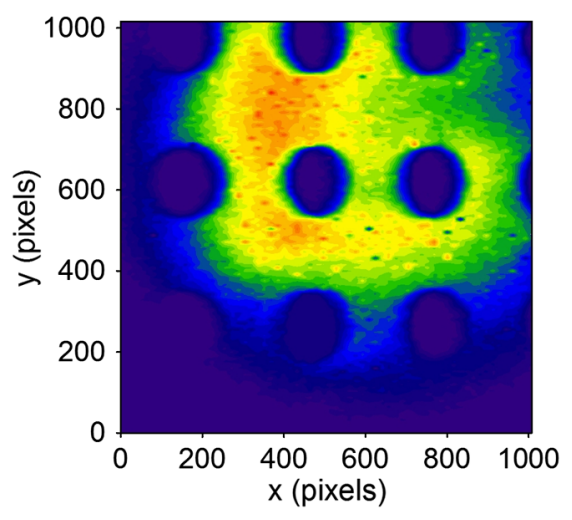

FIG. 8. Image of the calibration target. The increased vertical resolution at the expense of the horizontal is clearly visible.

was about $31 \%$ and the transmission width (FWHM) amounted to $2.6 \mathrm{~nm}(\Delta \lambda / \lambda=13 \%)$. The mirror normal was tilted by approximately $5.5^{\circ}$ with respect to the BTR emission direction, resulting in a spherical aberration contribution in the imaging process. The setup was aligned such that the aberration contribution affecting the vertical beam size was minimized at the expense of the horizontal resolution; see also Fig. 8. However, during the course of the experiment, beam images were recorded in the visible and in the EUV spectral regions under the same conditions, thus allowing a direct comparison. Such a relative beam size measurement is possible, even for the horizontal beam size, because spherical aberrations are independent of the radiation wavelength.

In order to calibrate the optical imaging system in the visible spectral region, an optical dot grid target with $0.5 \mathrm{~mm}$ dot spacing was used. At the beginning of the experiment, it was installed at the location of the BTR target and illuminated by a blue LED. During the optical system adjustment, the distance between the target and mirror was changed in order to obtain the best focusing. The resulting image is shown in Fig. 8. The horizontal and vertical calibration coefficients were not identical, namely, 1.69 and $1.43 \mu \mathrm{m}$ per CCD pixel, respectively.

\section{B. Measurements and results}

The accelerator was operated under the same conditions as in the previous experiment with a pulse duration of 0.8 and $3 \mathrm{~s}$ gaps. The average beam current was $2.4 \mathrm{nA}$. During the course of the experiment, different beam configurations with different beam sizes were used by changing the currents in the upstream quadrupole magnets.

For the initial alignment of the imaging setup with a beam, a $500 \mu \mathrm{m}$ thick LYSO:Ce scintillator crystal was utilized which was additionally mounted onto the target holder. The use of a scintillator instead of the BTR target was advantageous because of the high light yield and the isotropic emission. The beam image from the scintillator was found by changing the orientation of the spherical mirror. Afterwards, this orientation was kept fixed and the
BTR target was moved into the beam. BTR based beam images were obtained while scanning the target angle and the target-mirror distance.

For beam imaging, CCD images were recorded and accumulated over 100 accelerator pulses for measurements in the EUV spectral region and over 20 pulses for measurements with the BP filter. The images were taken for the same beam parameters while interchanging the filters in front of the CCD. In order to correct for background and $\mathrm{CCD}$ noise, additional CCD images were recorded with the beam switched on while the optical light path was blocked by a $1 \mathrm{~mm}$ thick aluminum plate. In a postprocessing step, these background images normalized to the number of shots were subtracted from the normalized CCD images with beam.

Figure 9 shows examples of beam images measured both in the visible region by using the $400 \mathrm{~nm}$ BP filter and in the EUV region by using the $1.3 \mu \mathrm{m}$ thick aluminum foil filter for the same electron beam parameters. The radiation intensity is expressed in CCD counts per pixel and normalized to a single accelerator pulse. By comparing the measurements in both wavelength regions, a clear beam
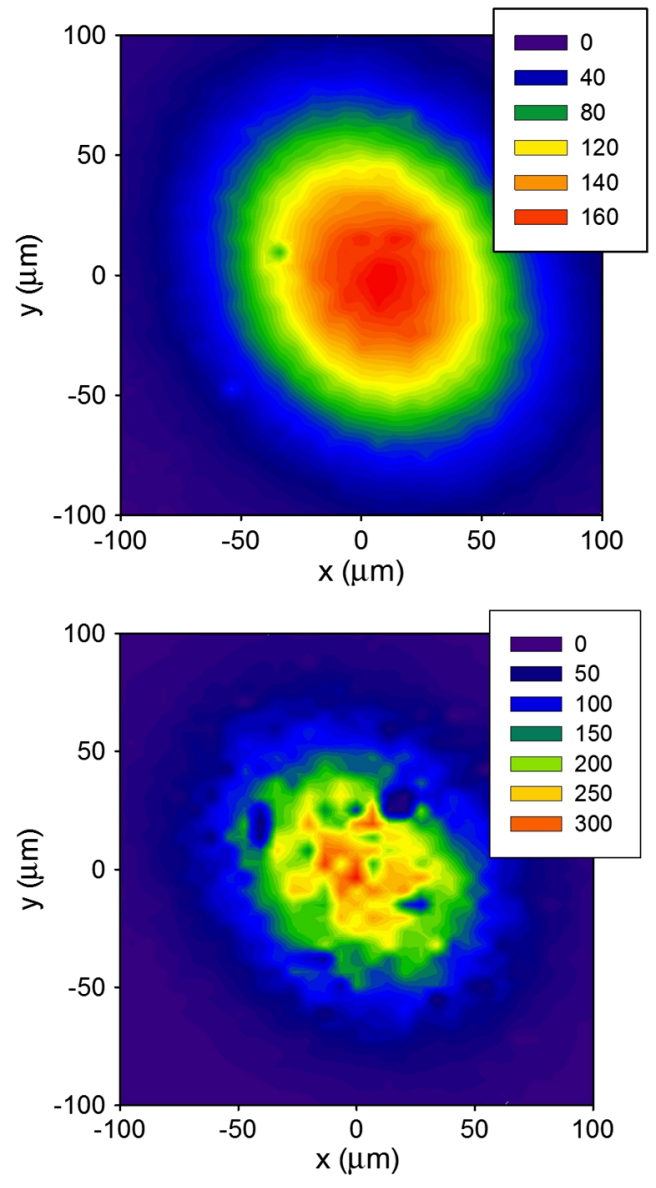

FIG. 9. Beam images obtained in the visible region by using the $400 \mathrm{~nm}$ BP filter (top) and in the EUV region by using the $1.3 \mu \mathrm{m}$ aluminum foil filter (bottom). 
spot in the EUV region can be recognized which is about a factor of 2 more intense than that in the visible part of the spectrum. In addition, dark spots in the EUV beam image are apparent which are caused by dirt which condensed onto the uncovered CCD chip during the cooldown of the detector. These foreign particles were transparent for visible light but had a strong absorption in the EUV region and could be removed after the experiment by using strong UV irradiation. Because of these spots, there is scope for interpretation in the comparison of the structure of the beam images. In Fig. 10, both horizontal and vertical projections of the beam images are plotted. To aid the comparison, the data are normalized to the peak value. The projections in both the visible and the EUV regions can be well described by a Gaussian distribution, despite the spots in the EUV image. From the fit it can be concluded that the beam image in the visible region is about $15 \%$ wider than that in the EUV region.

Figure 11 summarizes the comparison of measured beam sizes in the EUV and in the visible regions for different beam configurations. The sizes were obtained as rms values by fitting the projections with a Gaussian distribution. Fit errors in Fig. 11 are omitted because they are smaller than the drawn point size. As can be seen, beam sizes measured in the EUV spectral region are typically smaller than the corresponding values in the optical region. While the typical discrepancy is on the order of $15 \%$, for the small
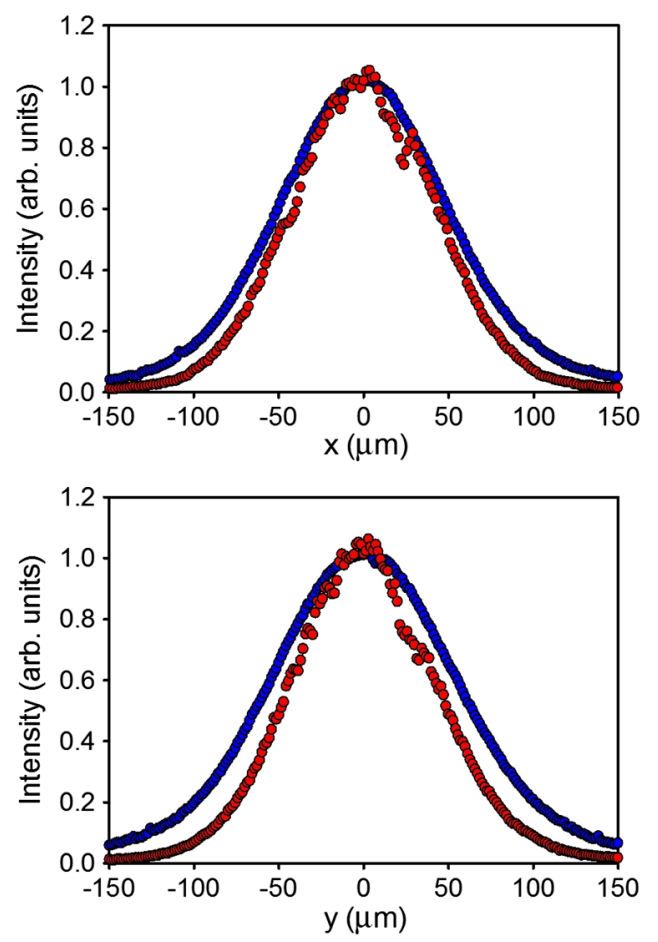

FIG. 10. Horizontal (top) and vertical (bottom) projections of beam images measured in the visible (blue dots) and the EUV (red dots) spectral region. To aid the comparison, the data are normalized to the peak value.

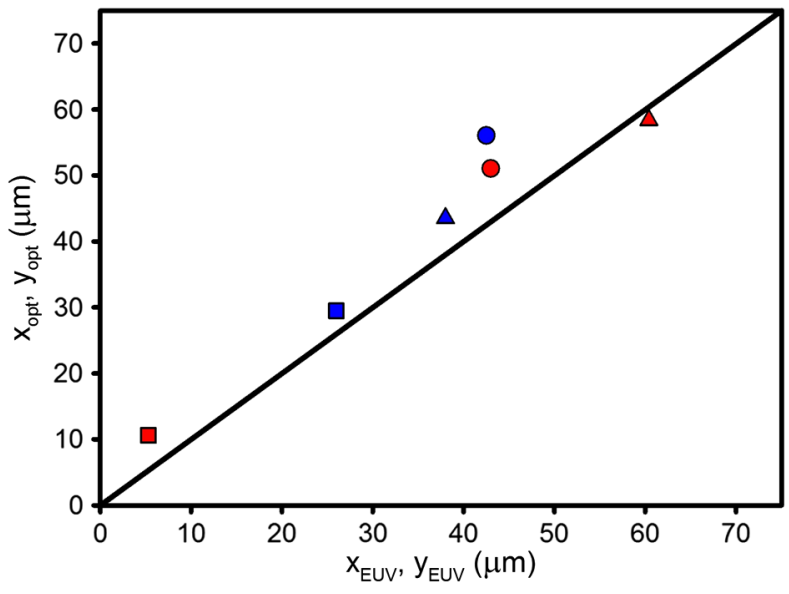

FIG. 11. Comparison of beam sizes obtained in the visible and the EUV spectral regions for different beam configurations. Circles, configuration No. 1; squares, configuration No. 2; triangles, configuration No. 3. Red points: Horizontal (rms) beam size; blue points: vertical beam size. The black solid line indicates the coincidence between the measurements in different spectral regions.

horizontal beam size it is $50 \%$. The observed discrepancy in the resulting beam sizes measured in different spectral regions cannot simply be explained by a smaller diffraction broadening contribution, i.e., the BTR PSF influence at smaller wavelengths. In our opinion, the most probable explanation for the observed additional broadening of the beam images is due to a small defocusing. The effect of defocusing and related wavelength-dependent PSF broadening is discussed in detail elsewhere [31]. There also might be an influence of aberrations in the optical system that results in additional broadening of the optical images. However, in our opinion, such an influence should manifest in both wavelength regions in the same way, because spherical aberrations are independent of wavelength.

\section{SUMMARY AND CONCLUSION}

This paper reports the first investigation of EUV backward transition radiation in view of transverse beam imaging. BTR in the EUV spectral region was clearly observed with promising intensity and could be used for beam diagnostic purposes.

In the first part of the experiment, the BTR angular distribution was investigated for different wavelength regions. The intensity of the measured radiation in the EUV spectral region was even higher than that measured in the visible region using a bandpass filter by a factor of about 2.5. However, this EUV yield is underestimated by the simple model presented in this paper. Nevertheless, the high EUV radiation yield observed is promising for applications in electron beam diagnostics.

The first electron beam profile measurements using EUV BTR made in the second part of the experiment are 
presented and compared to measurements with conventional BTR in the visible spectral region for identical beam parameters. Both methods result in the same beam shape and similar beam sizes. However, the beam sizes obtained via EUV imaging are typically $10 \%-15 \%$ smaller than those measured in the visible region. The expected reason for this observation is imperfections in the optical system such as a small defocusing, which will be optimized in a later stage of the experiment. In addition, the beam images recorded in the EUV region were deteriorated by spots on the CCD chip, caused most probably by dirt that condensed onto the cooled CCD chip. These particles could be removed only after the experiment in a complex cleaning procedure. Nevertheless, it was demonstrated that imaging in the EUV region works and that the achievable EUV intensity is sufficient to utilize it for beam diagnostics.

The results of the proof-of-principle experiment show that BTR in the EUV region is a promising candidate for an improvement of conventional optical transverse beam profile monitors in view of better resolution and the possibility to avoid coherent effects in the radiation generating process.

As a next step, it is planned to demonstrate that beam imaging based on EUV BTR may be considered as an efficient technique to overcome the problem of coherent optical radiation and to improve the resolution in view of submicron beam size imaging. For this purpose, a new aberration-free optical system will be developed and tested which allows absolute beam size measurements in the EUV or even soft x-ray region with submicron resolution.

\section{ACKNOWLEDGMENTS}

We express our gratitude to A. Delfs (DESY) for valuable contributions in the construction of the beam imaging experiment and to the Institute of Nuclear Physics (Mainz University) and DESY for help and support during the course of the experiments. We thank C. Laubis and F. Scholze (PTB, Berlin) for the reflectivity measurements of the multilayer coated mirror. We also express our gratitude to M. Lomperski (DESY) for careful proofreading of the manuscript. The work was partially supported by the Russian Ministry of Education and Science within the program "Nauka" Grant No. 3.709.2014/K.

[1] R. B. Fiorito and D. W. Rule, AIP Conf. Proc. 319, 21 (1994).

[2] M. Ross, S. Anderson, J. Frisch, K. Jobe, D. McCormick, B. McKee, J. Nelson, T. Smith, H. Hayano, T. Naito, and N. Terunuma, AIP Conf. Proc. 648, 237 (2002).

[3] H. Loos, R. Akre, A. Brachmann, F.-J. Decker, Y. T. Ding, D. Dowell, P. Emma, J. C. Frisch, A. Gilevich, G. R. Hays, P. Hering, Z. Huang, R. H. Iverson, C. Limborg-Deprey, A. Miahnahri, S. Molloy, and H.-D. Nuhn, in Proceedings of the Thirtieth Free Electron Laser Conference, Gyeongju,
South Korea (JACoW, Gyeongju, 2008), THBAU01, p. 485.

[4] S. Wesch, C. Behrens, B. Schmidt, and P. Schmüser, in Proceedings of the 31st International Free Electron Laser Conference (FEL 09), Liverpool, UK (STFC Daresbury Laboratory, Warrington, 2009), p. 619.

[5] XFEL Technical Design Report No. DESY 2006-097, http://xfel.desy.de.

[6] G. Kube, C. Behrens, and W. Lauth, in Proceedings of the International Particle Accelerator Conference, Kyoto, Japan (ICR, Kyoto, 2010), MOPD088, p. 906.

[7] M. Yan, C. Behrens, Ch. Gerth, G. Kube, B. Schmidt, and S. Wesch, in Proceedings of the 10th European Workshop on Beam Diagnostics and Instrumentation for Particle Accelerators, Hamburg, Germany (DESY, Hamburg, 2011), TUPD59, p. 440.

[8] G. Kube, C. Behrens, Ch. Gerth, B. Schmidt, W. Lauth, and M. Yan, in Proceedings of the 3rd International Particle Accelerator Conference, New Orleans, LA, 2012 (IEEE, Piscataway, NJ, 2012), WEOAA02, p. 2119.

[9] C. Behrens, Ch. Gerth, G. Kube, B. Schmidt, S. Wesch, and M. Yan, Phys. Rev. ST Accel. Beams 15, 062801 (2012).

[10] Ch. Wiebers, M. Holz, G. Kube, D. Nölle, G. Priebe, and H.-Ch. Schröder, in Proceedings of the Second International Beam Instrumentation Conference, Oxford, United Kingdom, 2013 (JACoW, Oxford, 2013), WEPF03, p. 807.

[11] H. Loos, in Proceedings of the Third International Beam Instrumentation Conference, Monterey, CA, 2014 WEIXB1.

[12] B. Walasek-Höhne and G. Kube, in Proceedings of the 10th European Workshop on Beam Diagnostics and Instrumentation for Particle Accelerators, Hamburg, Germany, 2011 (DESY, Hamburg, 2011), WEOB01, p. 553.

[13] B. Walasek-Höhne, C. Andre, P. Forck, E. Gütlich, G. Kube, P. Lecoq, and A. Reiter, IEEE Trans. Nucl. Sci. 59, 2307 (2012).

[14] M. Castellano and V. A. Verzilov, Phys. Rev. ST Accel. Beams 1, 062801 (1998).

[15] A.P. Potylitsyn, in Advanced Radiation Sources and Applications, edited by H. Wiedemann (Springer, Dordrecht, 2006), p. 149.

[16] D. Xiang and W.-H. Huang, Nucl. Instrum. Methods Phys. Res., Sect. A 570, 357 (2007).

[17] G. Kube, TESLA-FEL Report No. 2008-01, 2008.

[18] P. Karataev, A. Aryshev, S. Boogert, D. Howell, N. Terunuma, and J. Urakawa, Phys. Rev. Lett. 107, 174801 (2011).

[19] L. G. Sukhikh, S. Yu. Gogolev, and A. P. Potylitsyn, Nucl. Instrum. Methods Phys. Res., Sect. A 623, 567 (2010).

[20] L. G. Sukhikh, D. Krambrich, G. Kube, W. Lauth, Yu. A. Popov, and A. P. Potylitsyn, in Proceedings of SPIE The International Society for Optical Engineering. EUV and X-Ray Optics: Synergy Between Laboratory and Space II, Prague, Czech Republic, 8076, 80760G (SPIE, Bellingham, WA, 2011).

[21] L. G. Sukhikh, D. Krambrich, G. Kube, W. Lauth, Yu. A. Popov, and A. P. Potylitsyn, in Proceedings of the 10th European Workshop on Beam Diagnostics and Instrumentation for Particle Accelerators, Hamburg, Germany, 2011 (DESY, Hamburg, 2011), WEOA02, p. 544. 
[22] L. G. Sukhikh, S. Bajt, G. Kube, Yu. A. Popov, A. P. Potylitsyn, and W. Lauth, in Proceedings of the 3rd International Particle Accelerator Conference, New Orleans, LA, 2012 (IEEE, Piscataway, NJ, 2012), MOPPR019, p. 819.

[23] D. V. Karlovets and A. P. Potylitsyn, Phys. Lett. A 373, 1988 (2009).

[24] R. Manzke, Phys. Status Solidi (b) 97, 157 (1980).

[25] B. L. Henke, E. M. Gullikson, and J. C. Davis, At. Data Nucl. Data Tables 54, 181 (1993); http://henke.lbl.gov/ optical_constants/.

[26] A. Jankowiak, Eur. Phys. J. A 28, 149 (2006).

[27] http://www.incoatec.de.
[28] http://www.andor.com/scientific-cameras.

[29] http://www.e2v.com/products-and-services/high-performanceimaging-solutions/imaging-solutions-cmos-ccd-emccd/ datasheets/.

[30] G. Kube, H. Backe, H. Euteneuer, A. Grendel, F. Hagenbuck, H. Hartmann, K. H. Kaiser, W. Lauth, H. Schöpe, G. Wagner, Th. Walcher, and M. Kretzschmar, Phys. Rev. E 65, 056501 (2002).

[31] G. Kube, S. Bajt, Yu. A. Popov, A. P. Potylitsyn, L. G. Sukhikh, and W. Lauth, in Proceedings of the 4th International Particle Accelerator Conference, IPAC2013, Shanghai, China, 2013 (JACoW, Shanghai, China, 2013), MOPME010, p. 488. 